\title{
Investigating the Effectiveness of Tele-Counselling Psychological Intervention on the Perinatal Mental Health During the Outbreak of COVID-19: A Randomized Trial
}

\section{Sahebeh Dadshahi}

Kerman University of Medical Sciences

Atefeh Ahmadi ( $\nabla$ atefeahmadi59@gmail.com )

Kerman University of Medical Sciences

Masumeh Ghazanfarpour

Kerman University of Medical Sciences

Katayoun Alidousti

Kerman University of Medical Sciences

\section{Setareh Yousefi}

Kerman University of Medical Sciences

\section{Zahra Pakdel}

Kerman University of Medical Sciences

Masoumeh Nasrollahi

Kerman University of Medical Sciences

\section{Zahra kashfi}

Kerman University of Medical Sciences

\section{Fatemeh Esmaeilinejadhasaroeih}

Kerman University of Medical Sciences

\section{Rezvan Khaleghi}

Kerman University of Medical Sciences

\section{Parinaz Noormohammadi}

Kerman University of Medical Sciences

\section{Research Article}

Keywords: Psychological interventions, tele-counseling, mental health, Corona, perinatal period

Posted Date: February 25th, 2022

DOI: https://doi.org/10.21203/rs.3.rs-1113726/v1 
License: (c) (i) This work is licensed under a Creative Commons Attribution 4.0 International License. Read Full License 


\section{Abstract}

Design: This is a randomized trial with a control and an intervention group. The aim of this study was to investigate the effect of psychological interventions on women's mental health in the perinatal period duringsars-cov-2 pandemic.

Method: This study was performed on 60 women in the perinatal period (30 in the control group and 30 in the intervention group). The women in the intervention group were included in 6 weekly sessions of 90120 minutes of tele-counseling. Anxiety, depression, health anxiety and its dimensions were assessed by modified Hospital Anxiety-Depression Scale (HADS) and Short Health Anxiety Index (SHAI) before and after the intervention in both groups. Data were analyzed by SPSS-22 and Kolmogorov-Smirnov, ShapiroWilkes tests, one way-ANOVA and paired t-test, Kruskal-Wallis, Wilcoxon paired t-test and K-2.

Results: Health anxiety had a significant decrease in both dimensions (anxiety of being sick and negative consequences of disease $)(p=0.007, p=0.005)$ while depression and anxiety related to COVID-19 hadn't been changed significantly $(P=0.34$ and $p=0.92)$.

Conclusion: Psychological intervention by tele-counseling approach can be effective as a suitable therapeutic approach to reduce health anxiety in the perinatal period. Trial registration:

IRCT20170611034452N12. Trial Id: 50289, 12/20/2020 Keywords: Psychological interventions, telecounseling, mental health, Corona, perinatal period

\section{Introduction}

\section{Rationale \& background information:}

The COVID-19 pandemic has caused public panic and concern. The growing number of people with the COVID-19 is exacerbating fears among people. Misinformation, rumors, misunderstandings and the relative ignorance of the disease add to this anxiety. Travel bans, some executive regulations, quarantine, and the closure of many programs and festivals increase feelings of hopelessness and fear in the general population. The risk of COVID-19 during pregnancy may be higher than the general population. Many of the consequences of COVID-19 on pregnancy outcomes are not detected but complications of other coronaviruses e.g., SARS, make pregnant woman with COVID-19 being considered potentially vulnerable to negative consequences. Impact of pregnancy on the respiratory system, immune system, coagulation and cardiovascular function may have influence on COVID-19 disease progression. In addition, its effects on fetal growth and development, labor, postpartum and neonatal health is somehow unclear. Anxiety causes the mother to respond inappropriately to the fetus during pregnancy and reduces the neonatalmaternal attachment. Therefore, it is necessary to do early and hard interventions in coping with these prenatal anxieties. Non-pharmacological methods are one of the most effective ways to cope with anxiety. studied the effect of tele-counselling on the mental health of hospital and infirmary personnel during the COVID-19 epidemic and showed that the anxiety related to the COVID-19 and the anxiety related to the likelihood of developing the COVID-19 disease reduced in the intervention group 
significantly compared with the control group $(p=0.001,0.001)$. So far, no interventional study has used the effect of tele-counselling on maternal mental health during the prenatal period in Iran. Regarding the importance of the effect of mental problems on mothers, fetuses and infants in this period, this study was conducted to investigate the effect of this method on mental health of pregnant women covered by health infirmaries during the coronavirus pandemic.

\section{Study goals and objectives}

General goal: Effectiveness' determination of psychological interventions on the mental health of women referred to hospital and health centers affiliated to the Kerman University of Medical Science in pregnancy, delivery and the postpartum period in the Coronavirus epidemic in 2020.

Specific goals:

1-Determining and comparing depression of married women referring to hospitals and clinics of Kerman University of Medical Sciences during pregnancy, childbirth and postpartum during the outbreak of coronavirus before and after counseling in the intervention group

2-Determining and comparing depression of married women referring to hospitals and clinics of Kerman University of Medical Sciences during pregnancy, childbirth and postpartum during the outbreak of coronavirus before and after counseling in the control group

3-Comparison of Depression in Married Women Referred to Hospitals and Clinics of Kerman University of Medical Sciences during Pregnancy, Childbirth and Postpartum During Coronavirus Outbreak Before and After Counseling Between Intervention and Control Groups

4- Determining and comparing the anxiety of married women referring to hospitals and clinics of Kerman University of Medical Sciences during pregnancy, childbirth and postpartum in the period of coronavirus outbreak before and after counseling in the intervention group

5- 4- Determining and comparing the anxiety of married women referring to hospitals and clinics of Kerman University of Medical Sciences during pregnancy, childbirth and postpartum in the period of coronavirus outbreak before and after counseling in the control group

6- Comparison of Anxiety of Married Women Referred to Hospitals and Clinics of Kerman University of Medical Sciences during Pregnancy, Childbirth and Postpartum During the Coronavirus Outbreak before and after consultation between the intervention and control groups

\section{Methods}

Trial design: Randomized trial parallel interventional research, with pre-test and post-test, control and intervention design and simple random sampling with a sample size of 60 people (allocation ratio:1/1). Women satisfied to participate in the study will be randomly divided into intervention and control groups. 
Randomization in this study is a simple randomization. It is a personal randomization unit. Layering (strata) is not done.

The methods were carried out in accordance with the Declaration of Helsinki. Trial registration: IRCT20170611034452N12. Registration number: 50289, date of first registration: 12/20/2020.

This project was carried out after the approval and acquisition of the code of ethics (IR.KMU.REC.1399.134) from Kerman University of Medical Sciences.

Participants: Women (intervention $(n=30)$ and control $(n=30)$ ) in perinatal period who were under the affiliation of health care centers of Kerman University of Medical Sciences in May and June 2020 and fill informed consent forms. Inclusion criteria: satisfaction to participate in the study, being in a time period of 28 weeks of pregnancy to 28th of postpartum day for women referring for postpartum services, not receiving psychiatric medication, not attending in counselling sessions at the same time of study period. Exclusion criteria were exposure to severe acute stress during the study (still birth, neonatal death or eclampsia...), not attending in more than one counseling session.

Statistical consultant of the study generated the random allocation sequence, data collectors of the research enrolled participants and assigned participants to interventions:

\section{Intervention}

The intervention group will attend 6 psychological counselling group sessions through virtual networks such as WhatsApp or Skype for 90 to 120 minutes. Statistical consultant of the research generated the random allocation sequence, data collectors of the team enrolled participants, and assigned participants to interventions, too. Participants of the intervention and control groups were selected based on the day of referral, so that those pregnant clients who referred to health centers on even days were assigned in the intervention group and those pregnant clients who referred to health centers on odd days were assigned in the control group, and this process was reversed next week. Individuals were also assured that all their information was confidential, and that the results would be outlined generally. The participants were then asked to complete the questionnaires as a pre-test. Both groups completed the questionnaire as a posttest after the counseling sessions. Table 1 shows briefly the contents of the sessions. 
Table 1

Content of psychological intervention sessions in the form of tele counseling

\section{Session Content}

1 Training of how to prevent the COVID-19 and provision of information of health according to their condition of women, dysfunctional cognitions related to COVID-19, muscle relaxation technique, conscious eating technique, assignment

2 Reviewing assignments, defining mindfulness and its benefits in coping with COVID-19 anxiety, mindful breathing and senses, expressing personal boundaries and limitations, assignments

3 Review of assignments, familiarity with the symptoms of stress and anxiety, introduction of cognitive errors related to COVID-19 from Ellis and Beck's perspectives, training of ways to overcome pregnancy and postpartum fatigue, yoga and aerobic exercise to increase respiratory capacity, assignments

4

Review of assignments, sitting meditation with focus on breathing, body sounds and thoughts (four-dimensional meditation), definition of autopilot mind, familiarity with coping styles in the face of stressors, assignments.

5

Reviewing assignments, re-discussing cognitive errors, disadvantages and advantages of worries and fears, promoting family relationships and making love to the fetus and neonate, defining the pleasant and unpleasant life events, and ways to change feelings and attitudes towards events, assignments.

6

Accepting conditions and commitments by relying on self-care, being at the present (quitting daydreaming), discussing one's fears about one's vulnerability to disease, doing a body scan, reviewing and concluding.

The control group received no intervention and the participants of this group were put on a waiting list.

Outcomes: Data collection tools included demographic and midwifery questionnaire (Appendix 1), Health Anxiety Questionnaire (HAQ) (Appendix 2) and Hospital Anxiety and Depression Scale (HADS) (Appendix 3). The demographic-midwifery questionnaire was about demographic characteristics and midwifery records. Salkovskis and Warwick first developed the HAQ in 1989. This questionnaire was based on the cognitive model of health anxiety and hypochondriasis. Salkovskis and Warwick (2002) again developed the 18-item short self-report version of this questionnaire. Each item has four choices, which includes one's description of the components of health and disease in form of declarative sentences and the subject should choose one of the sentences that best describes him/her. The minimum score in this questionnaire is zero and the maximum score is 54. The HADS consists of two parts: demographic variables and the Hospital Anxiety and Depression Scale by Zigmond \& Snaith (1983). This four-item questionnaire has been designed to assess mood changes, especially anxiety and depression. Scores of depression and anxiety subscales of the HADS include zero-seven (normal), 8-10 (mild), 11-14 (moderate) and 15-21 (severe). For the reliability of the questionnaire to be determined, Cronbach's alpha method was used on 167 patients with cancer, which was 0.78 for anxiety and 0.86 for depression (Montazeri et al., 2003). Its validity was $0.47-0.83$ for anxiety and $0.48-0.86$ for depression by using Beck Depression Inventory. Concurrent validity of the depression subscale in the HADS and Beck Depression Inventory is 0.8 at the significance level of 0.0001 . The internal consistency of the 
questionnaire is 0.82 for the anxiety subscale and 0.83 for the depression subscale by Cronbach's alpha method, which was performed on 745 cancer patients (16).

Women filled the instruments before the first session and three weeks after the last session. The impact of counseling was compared in the two groups. Psychological intervention can reduce the anxiety related to likelihood of developing the disease and anxiety of the negative disease consequences. Therefore, this approach can be effective in reducing women's anxieties in this stressful period.

\section{Sample size:}

However, regarding probable dropout and since the minimum number of participants required for parametric testing is 30 individuals in each group, the final sample size was considered 60 (30 in each group) (15).

$$
n=\frac{\left(s_{1}^{2}+s_{2}^{2}\right)^{2}\left(z_{1-\frac{\alpha}{2}}+z_{1-\beta}\right)^{2}}{\left(\bar{x}_{1}-\bar{x}_{2}\right)^{2}}
$$

\section{Randomization}

For the division of them into two groups of intervention and control, statistical software will used. The tool used in randomization is Randomizer software (www.randomizer.org) which also performs random sequences.

\section{Allocation concealment mechanism}

does not apply to this counselling intervention. The post-test will be offered to the two groups after the sessions.

\section{Implementation}

Using random-numbers table the random allocation sequence was generated, The staff of health centers and hospital enrolled participants, and randomly assigned participants to the intervention and control groups.

\section{Blinding}

not blinded to treatment allocation but Those assessing outcomes were blinded by not mentioning the name of the groups in the SPSS file sent for analysis.

Description of the similarity of interventions: not applicable

\section{Statistical method}


Data were analyzed by SPSS 22, Kolmogorov-Smirnov, Shapiro Wilkes, one-way analysis of variance, paired t-test, Kruskal-Wallis, Wilcoxon paired t- and Chi-square tests. After collecting the sample data, the study data were classified into intervention and control groups and entered into SPSS 22 software. For demographic and descriptive data, central indices and dispersion are calculated and presented in the form of frequency tables and appropriate graphs. For data analysis, for quantitative data, depending on the type of distribution, appropriate parametric and non-parametric tests will be used to check the normality before analyzing the distribution of the consulting and control group in terms of normality by Kolmogorov-Smirnov and Shapiro tests. Wilkes is measured. If normal, one-way analysis of variance and paired t-test are used, and if the data distribution is not normal, the non-parametric equivalent of one-way analysis of variance, Kruskal-Wallis, and Wilcason paired t-test will be used. It is used to calculate the significant relationship between the relevant variables with a significance level of 0.05 and a statistical power of $80 \%$. For qualitative variables, chi-square test is used to examine the significant relationship.

\section{Results}

Sixty women were studied. Table 2 shows the demographic information of both groups based on chisquare test before the intervention. The mean age of most of the women was $29.40 \pm 5.7$ years, half of the women had middle, high school and academic education and most of them were housewives. Chisquare test showed no significant difference between the two groups in demographic variables.

Therefore, the groups were homogeneous in terms of maternal age $(P=0.94)$, living place $(P=0.57)$, education ( $P=0.94)$, occupation $(P=0.47)$, number of deliveries $(P=0.2)$, type of delivery $(P=0.96)$, and number of abortions $(P=0.87)$. The t-test in Table 3 showed a significant decrease in the scores of anxiety, the likelihood of developing the disease and anxiety of the negative disease consequences $(P=$ 0.007 and $P=0.005$, respectively), but the hospital anxiety and depression index did not change $(P=0.34$ and $P=0.92$, respectively). 
Table 2

Demographic and clinical variables of individuals in the two groups of intervention and control

\begin{tabular}{|c|c|c|c|c|}
\hline Variables & & Intervention & Control & P-value \\
\hline Age & & $29.4 \pm 5.8$ & $29.3 \pm 5.7$ & 0.94 \\
\hline \multirow[t]{2}{*}{ City } & Kerman & $28(52.8)$ & $25(47.2)$ & \multirow[t]{2}{*}{0.57} \\
\hline & Others & $12(52.8)$ & $11(47.8)$ & \\
\hline \multirow[t]{5}{*}{ Education } & Uneducated & 0 & 0 & \multirow[t]{5}{*}{0.94} \\
\hline & Elementary & 0 & 0 & \\
\hline & Middle school & $3(50)$ & $3(50)$ & \\
\hline & High school & $16(50)$ & $16(50)$ & \\
\hline & University & $17(44.7)$ & $21(55.3)$ & \\
\hline \multirow[t]{3}{*}{ Job } & Housewife & $31(50)$ & $31(50)$ & \multirow[t]{3}{*}{0.47} \\
\hline & Employed & 8 (61.5) & $5(38.5)$ & \\
\hline & Self-employed & $1(100)$ & 0 & \\
\hline \multirow[t]{3}{*}{ Delivery No. } & 0 & $14(53.8)$ & $12(46.2)$ & \multirow[t]{3}{*}{0.2} \\
\hline & 1 & $19(55.9)$ & $12(44.1)$ & \\
\hline & $>1$ & $7(53.6)$ & $9(46.3)$ & \\
\hline \multirow[t]{4}{*}{ Delivery type } & None & $13(52)$ & $12(48)$ & \multirow[t]{4}{*}{0.96} \\
\hline & Vaginal & $17(51.5)$ & $16(48.5)$ & \\
\hline & Cesarean & $10(6.55)$ & $8(44.4)$ & \\
\hline & Others & 0 & 0 & \\
\hline \multirow[t]{2}{*}{ Abortion } & No & 33 (52.4) & $30(47.6)$ & \multirow[t]{2}{*}{0.87} \\
\hline & Yes & $7(58.3)$ & $6(41.6)$ & \\
\hline \multirow[t]{3}{*}{ Disease history } & None & $31(52.5)$ & $28(47.5)$ & \multirow[t]{3}{*}{0.62} \\
\hline & Autoimmune & $1(100)$ & 0 & \\
\hline & Others & $8(50)$ & $8(50)$ & \\
\hline Medication dosage & None & $32(52.5)$ & $29(47.5)$ & 0.19 \\
\hline
\end{tabular}




\begin{tabular}{|c|c|c|c|c|}
\hline \multicolumn{2}{|l|}{ Variables } & \multirow{2}{*}{$\begin{array}{l}\text { Intervention } \\
5(41.7)\end{array}$} & \multirow{2}{*}{$\begin{array}{l}\text { Control } \\
7(58.3)\end{array}$} & \multirow[t]{3}{*}{ P-value } \\
\hline & Routine & & & \\
\hline & Specific & $3(100)$ & 0 & \\
\hline \multirow[t]{3}{*}{ Exercise } & Yes & $1(50)$ & $1(50)$ & \multirow[t]{3}{*}{0.81} \\
\hline & No & $24(55.8)$ & $19(44.2)$ & \\
\hline & Sometimes & $15(48.4)$ & $16(51.6)$ & \\
\hline \multirow[t]{3}{*}{ Stress } & No & $38(60.3)$ & $25(39.7)$ & \multirow[t]{3}{*}{0.003} \\
\hline & Died & $1(8.3)$ & $11(91.7)$ & \\
\hline & Surgery & $1(100)$ & 0 & \\
\hline \multirow[t]{3}{*}{ Type of stress } & COVID-19 & $4(44.4)$ & $5(55.6)$ & \multirow[t]{3}{*}{0.18} \\
\hline & Others & $6(35.3)$ & $11(64.7)$ & \\
\hline & None & $30(60)$ & $20(40)$ & \\
\hline
\end{tabular}

The aP-value compared with the mean difference (before and after) between control and intervention P-value between control group and intervention group at baseline 
Table 3

Comparison of depression and anxiety related to coronavirus, anxiety related to the likelihood of developing disease and anxiety of negative consequences in control $(n=44)$ and intervention $(n=51)$

\begin{tabular}{|c|c|c|c|c|}
\hline Variables & $\begin{array}{l}\text { Mean } \pm \text { SD before } \\
\text { intervention }\end{array}$ & $\begin{array}{l}\text { Mean } \pm S D \text { after } \\
\text { intervention }\end{array}$ & $\begin{array}{l}\text { Pre-and post-intervention mean } \\
\text { (SD) difference }\end{array}$ & $\begin{array}{l}\mathrm{P} \text { - } \\
\text { value }\end{array}$ \\
\hline \multicolumn{5}{|c|}{ Hospital anxiety related to the COVID-19 } \\
\hline Intervention & $18.2 \pm 2.57$ & $18.6 \pm 2.94$ & \multirow[t]{2}{*}{0.76} & \multirow[t]{2}{*}{0.34} \\
\hline Control & $18.4 \pm 3.13$ & $18.1 \pm 4.77$ & & \\
\hline \multicolumn{5}{|c|}{ Hospital depression related to COVID-19 } \\
\hline Intervention & $16.02 \pm 2.3$ & $12.4 \pm 1.97$ & \multirow[t]{2}{*}{0.98} & \multirow[t]{2}{*}{0.92} \\
\hline Control & $15.16 \pm 1.97$ & $12.94 \pm 1.88$ & & \\
\hline \multicolumn{5}{|c|}{ Anxiety related to likelihood of developing COVID-19 } \\
\hline Intervention & $30.1 \pm 6.5$ & $27.9 \pm 6.2$ & \multirow[t]{2}{*}{2.6} & \multirow[t]{2}{*}{0.007} \\
\hline Control & $28.2 \pm 6.9$ & $28.7 \pm 6.5$ & & \\
\hline \multicolumn{5}{|c|}{ Anxiety related to negative disease consequences } \\
\hline Intervention & $5.2 \pm 1.9$ & $4.7 \pm 1.6$ & \multirow[t]{2}{*}{0.91} & \multirow[t]{2}{*}{0.005} \\
\hline Control & $5.2 \pm 2.2$ & $5.6 \pm 2.1$ & & \\
\hline
\end{tabular}

\section{Discussion}

The corona pandemic has greatly affected the mental health of women. Since a high level of prenatal anxiety is a predictor of postpartum depression (6)and is associated with vomiting, preeclampsia, weight loss, preterm labor, low birth weight, decreased Apgar score, and neonatal abnormalities (7-9), it is necessary to perform early and hard interventions to cope with these prenatal anxieties (6) Nonpharmacological methods are one of the most effective ways to cope with anxiety (17). The present study aimed to investigate the effect of tele-counselling on prenatal mental health during the coronavirus outbreak in Kerman, which showed the significant effect of intervention on health anxiety. Although such an intervention affected the hospital anxiety and depression index, it was not significant.

Some limitations include low generalizability of study results due to the limited research population, nonparticipation in the intervention or excessive absences (in more than one counseling session), failure to perform tasks assigned in previous sessions and problems related to how to communicate with research units and obtain their consents and ensure the confidentiality of information. Owing to the fact that the results of the present study are related to the city of Kerman, it is suggested that similar studies be conducted in other places to understand the psychological consequences following the global outbreak of corona. 
The results of this study are consistent with the studies of Duan et al. (2020), Huang et al. (2020) and Zhang et al. (2020) and Ghazanfarpour et al. (2020) (18-20). This consistency can indicate the effects of psychological interventions on anxiety. Safar Ali Nejad et al. (2018) examined the effect of group behavioral-psychological counseling on depression during pregnancy. They showed a statistically significant difference in between- and within-group mean scores of depression syndrome (21). It is contrary to our study because depression has not changed after the intervention.

Fedakar et al. (2019) evaluated the effectiveness of life review therapy with a share perspective in improving family functioning and communication patterns of pregnant women in the Kermanshah earthquake crisis. They showed that the therapy significantly improved communication patterns (subscales of cross-constructiveness, expectation/withdrawal and mutual avoidance) and overall family functioning of the experimental group compared with the control group (22). This study supports the present study because the intervention was done on pregnant women and had positive effects.

One study showed the effect of psychological interventions on improving the mental health of individuals in this critical period (18). Huang et al. (2020) studied the effect of dialectical behavior therapy-based psychological intervention on women suffering from the COVID-19 in late pregnancy and early postpartum. Results showed that such an intervention was more useful for individuals with negative thoughts and moods. This method is both flexible and necessary for women who suffer from COVID-19 in late pregnancy and early postpartum (19). A similar study on public during the COVID-19 outbreak showed the positive effect of intervention on those under a lot of stress (19). In addition, tele-counselling showed a positive effect on anxiety of the medical staff, and coronavirus-related anxiety and the anxiety related to the likelihood of developing disease significantly decreased in the intervention group compared with the control group ( $p=0.001$ and 0.001$)$ (15).

One study showed both telephone and in-person counselling method, in comparison with usual care were efficacious the same (23). In a randomized trial compared telephone counseling with in-person counseling showed the first one led to lower effect but can be effectively used to increase reach and access without long-term adverse psychosocial consequences. Test uptake was lower for telephone counseling than in-person counseling. Uptake was lower for urban compared with rural dwellers in both counseling methods. Telephone counseling was noninferior to in-person counseling for all psychosocial and informed decision-making outcomes, at the 1-year follow-up. (24).

However, the interventions failed to affect the hospital anxiety and depression index because according to the World Health Organization, people are afraid of developing the COVID-19 disease during the outbreak of coronavirus, so it has designed a series of guidelines that are effective for all different classes and occupations (25). Reliable training reduces the rate of depression, and such training has had its effect before our intervention (26).

Furthermore, one of the tools of social support is information support, which is provided through the media, and a study has shown that social support reduces the incidence of depression (32). (27)The simplest explanation for high score of the depression is that participants fail to follow up exercises. Like 
other studies $(28,29)$ controlling unwanted variables and the short interval between the post-test and the follow-up period can be effective.

Unlike this group intervention, individual training makes it easier to follow up the participants, identify and control the confounding factors. Online education was used due to the spread of coronavirus, which could reduce the quality of work. According to Bagheri Majd et al. (2014), the five principles of education were less observed in virtual education compared with face-to-face one (30).

A number of women in the postpartum period experience a series of symptoms, including emotional despair following the excitement and fears during pregnancy and childbirth, early postpartum discomforts (puerperium), fatigue related to insomnia, anxiety related to abilities to care for the neonate, and concerns about the body appearance (31)which may cause the exercises not to be persistent. According to IRNA, people are afraid of going to medical centers during this period, the number of referrals has decreased, so mild mental disorders might not have been identified, which could affect the training.

According to the latest statistics on the number of patients with coronavirus on August 13, the number of victims with the COVID-19 disease has reached 747,258 in the world.

\section{Conclusion}

Psychological intervention can reduce the anxiety related to likelihood of developing the disease and anxiety of the negative disease consequences. Therefore, this approach can be effective in reducing women's anxieties in this stressful period.

\section{Declarations}

- Ethics approval and consent to participate

All methods were carried out in accordance with relevant guidelines and regulations. This study was conducted by obtaining the code of ethics (IR.KMU.REC.1399.134) from the dean of research of Kerman University of Medical Sciences, permission of the head and director of hospitals and infirmaries affiliated to Kerman University of Medical Sciences and informed consent from participants.

All methods were carried out in accordance with relevant guidelines and regulations. All experimental protocols were approved by a full name and institutional and licensing committee.The purpose of this study is to promote women's mental health during pregnancy, childbirth and postpartum.

The target group is pregnant women from 28 weeks to delivery 28 days after delivery.

Participation in the study is completely voluntary and cancellation at any stage of the research will not prevent you from receiving services and care. Informed consent was obtained from all subjects 
Conduct counseling sessions for 30-45 minutes, which will be completed during 6 sessions, which are performed twice a week for up to 3 weeks, before and after the end of the questionnaire sessions, which will be measured by comparing our intended questionnaire.

In this study, no physical or psychological risk will be threatened.

Because the role of the mother in society is a fundamental role, the mental health of a mother will affect the whole society. The goal of promoting mental health goes back not only to the individual but to society as a whole.

Because these sessions will be sent online via WhatsApp, no shipping costs will be charged and no fees will be charged to participants.

In addition, your identity information will not be recorded in the questionnaire, but this information will remain completely confidential.

Research findings will be published in journals and articles.

I, the owner of Dadshahi, who is conducting this research, am answering your questions

Phone number - WhatsApp 09104496351

Email sahebeh68.dadshahi@gmail.com

Withdrawal at any stage of the research is completely optional

- Consent for publication

All the participants provided informed consent to publish data without their names

- Availability of data and materials

The datasets used and/or analysed during the current study available from the corresponding author on reasonable request.Data cannot be deposited publicly as these collaborative data originate from multiple health clinics and hospitals with different legal frameworks.

- Competing interests:

The authors declare that they have no competing interests.

- Funding:

Kerman university of medical sciences, 99000034.

- Authors' contributions: 
S.D.: contributed to writing the main manuscript

K.A.: contributed to writing the main manuscript

A.A.: contributed to the study conception and design

M.G.: analysis

S.Y.: contributed to data gathering and holding counseling

Z.P.: contributed to data gathering and holding counseling

MN.: contributed to data gathering and holding counseling

Z.K.: contributed to data gathering and holding counseling

F.E.: contributed to data gathering and holding counseling

R.K.: contributed to data gathering and holding counseling

P.N.: contributed to data gathering and holding counseling

All authors read and approved the final manuscript.

- Acknowledgement

We would thank authorities of Razi School of Nursing and Midwifery in Kerman, managers and staff of infirmaries and hospitals affiliated to Kerman University of Medical Sciences and participants who helped us in this project.

\section{References}

1. Bao Y, Sun Y, Meng S, Shi J, Lu L. 2019-nCoV epidemic: address mental health care to empower society. The Lancet. 2020;395(10224):e37-e8.

2. Wastnedge EAN, Reynolds RM, van Boeckel SR, Stock SJ, Denison FC, Maybin JA, et al. Pregnancy and COVID-19. Physiological reviews. 2021 Jan 1;101(1):303-18. PubMed PMID: 32969772. Pubmed Central PMCID: PMC7686875. Epub 2020/09/25. eng.

3. Zhu H, Wang L, Fang C, Peng S, Zhang L, Chang G, et al. Clinical analysis of 10 neonates born to mothers with 2019-nCoV pneumonia. Translational pediatrics. 2020;9(1):51.

4. Stockman LJ, Lowther SA, Coy K, Saw J, Parashar UD. SARS during pregnancy, United States. 2004.

5. Calil VMLT, Krebs VLJ, Carvalho WBd. Guidance on breastfeeding during the Covid-19 pandemic. Revista da Associação Médica Brasileira. 2020;66(4):541-6.

6. Delavar Gavam S, Alizadeh Goradel J. The role of metacognitive beliefs and positive and negative affect in the Fear of childbirth of pregnant women with first experience. Iranian Journal of Nursing 
Research. 2014;9(3):10-8.

7. Khoursandi M, Vakilian K, Torabi Goudarzi M, Abdi M. Childbirth preparation using behavioralcognitive skill in childbirth outcomes of primiparous women. Journal of Babol University of Medical Sciences. 2013;15(4):76-80.

8. Akbarzadeh M, Toosi M, Zare N, Sharif F. effect of relaxation and attachment behaviors training on anxiety in first-time mothers in shiraz city, 2010: a randomized clinical trial (clinical trial article). 2013.

9. Toosi M, Akbarzadeh M, Zare N, Sharif F. Effect of attachment training on anxiety and attachment behaviors of first-time mothers. Journal of hayat. 2011;17(3):69-79.

10. Karamozan M, Askarizadeh G. Effectiveness of cognitive-behavioral stress management intervention on anxiety and depression during pregnancy. Journal of Kerman University of Medical Sciences. 2013;20(6):606-21.

11. Shobeiri F, Taravati-Javad M, Soltani F, Karami M. Effects of progressive muscle relaxation counseling on anxiety among primigravida women referred to health care centers in Hamadan. J Educ Community Health. 2015;2(2):1-9.

12. Rahimi F, Ahmadi M, Rosta F, Majd HA, Valiani M. Effect of relaxation training on pregnancy anxiety in high risk women. Safety Promotion and Injury Prevention. 2014;2(3):180-8.

13. JAYALAKSHMI R. tele-counseling for covid child survivor under hospital quarintine.

14. Ghazanfarpour M, Ashrafinia F, Zolala S, Ahmadi A, Jahani Y, Hosseininasab A. Investigating the Effectiveness of Tele-Counseling on Mental Health of Staff in Coronavirus Reference Clinics and Hospitals. Available at SSRN 3572898. 2020.

15. Karami K, Mardani A, SHAKERINEZHAD GA, Saki A. Effectiveness of a codified educational behavioral program on the mental health of pregnant women. 2015.

16. Muszbek K, Szekely A, Balogh ÉM, Molnár M, Rohánszky M, Ruzsa Á, et al. Validation of the Hungarian translation of hospital anxiety and depression scale. Quality of Life Research. 2006;15(4):761-6.

17. Saisto T, Toivanen R, Salmela-Aro K, Halmesmäki E. Therapeutic group psychoeducation and relaxation in treating fear of childbirth. Acta obstetricia et gynecologica Scandinavica. 2006;85(11):1315-9.

18. Duan L, Zhu G. Psychological interventions for people affected by the COVID-19 epidemic. The Lancet Psychiatry. 2020;7(4):300-2.

19. Huang J-W, Zhou X-Y, Lu S-J, Xu Y, Hu J-B, Huang M-L, et al. Dialectical behavior therapy-based psychological intervention for woman in late pregnancy and early postpartum suffering from COVID19: a case report. Journal of Zhejiang University Science B. 2020:1-6.

20. Zhang J, Wu W, Zhao X, Zhang W. Recommended psychological crisis intervention response to the 2019 novel coronavirus pneumonia outbreak in China: a model of West China Hospital. Precision Clinical Medicine. 2020;3(1):3-8. 
21. Safaralinezhad A, Oveisi S, Jourabchi Z. Effect of cognitive-behavioral group therapy on gestational depression: A clinical trial. The Iranian Journal of Obstetrics, Gynecology and Infertility. 2018;21(2):48-59.

22. Fedakar Davarani, Seifi, Kazemian. The effectiveness of life review therapy with a share perspective on improving family functioning and communication patterns of pregnant women in the Kermanshah earthquake crisis. Journal of Cognitive Analytical Psychology .; 10 (39): 39-50 [Persian].

23. Jarrett ME, Cain KC, Burr RL, Hertig VL, Rosen SN, Heitkemper MM. Comprehensive self-management for irritable bowel syndrome: Randomized trial of in-person versus combined in-person and telephone sessions. The American journal of gastroenterology. 2009;104(12):3004.

24. Kinney AY, Steffen LE, Brumbach BH, Kohlmann W, Du R, Lee J-H, et al. Randomized noninferiority trial of telephone delivery of BRCA1/2 genetic counseling compared with in-person counseling: 1year follow-up. Journal of Clinical Oncology. 2016;34(24):2914.

25. Organization WH. Mental health and psychosocial considerations during the COVID-19 outbreak, 18 March 2020. World Health Organization, 2020.

26. Farajzadeh Z, KeramatiNia F, Saadatjoo SA, Khazaie K. The Effect of Cognitive-Behavioral Teaching on Depression of Nurses in the Teaching Hospitals of Birjand. Journal of Nursing Education (JNE). 2019;7(6).

27. Salehi K, Mahmodifar Y. Relationship between social support and depression, anxiety in Hemodialysis patients. Iranian Journal of Nursing Research. 2014 May 10; 9 (1): 33-9. [Persian].

28. Bondolfi G, Jermann F, Van der Linden M, Gex-Fabry M, Bizzini L, Rouget BW, et al. Depression relapse prophylaxis with Mindfulness-Based Cognitive Therapy: replication and extension in the Swiss health care system. Journal of affective disorders. 2010;122(3):224-31.

29. Khanipour, Hamid, Borjali, Ahmad, Mohammadkhani, Parvaneh, Sohrabi. The effectiveness of mindfulness-based cognitive therapy on depressive and thought-provoking symptoms in people with a history of major depression: A single case study. Clinical Psychology Studies. 2013 Dec 22; 4 (13): 23-42 [Persian] .

30. Bagheri Majd R, Ghale'ei A, Mohajeran B, Sedghi Bokani N, Eslahi M. Comparison of Commitment to Pedagogy in E-Learning and Traditional Training in Iran Higher Education System. Education Strategies in Medical Sciences. 2014;7(4):221-7.

31. Cunningham F, Leveno K, Bloom S, Spong CY, Dashe J. Williams obstetrics, 24e: Mcgraw-hill; 2014.

\section{Figures}




\section{Checklist 2010 Flow Diagram}

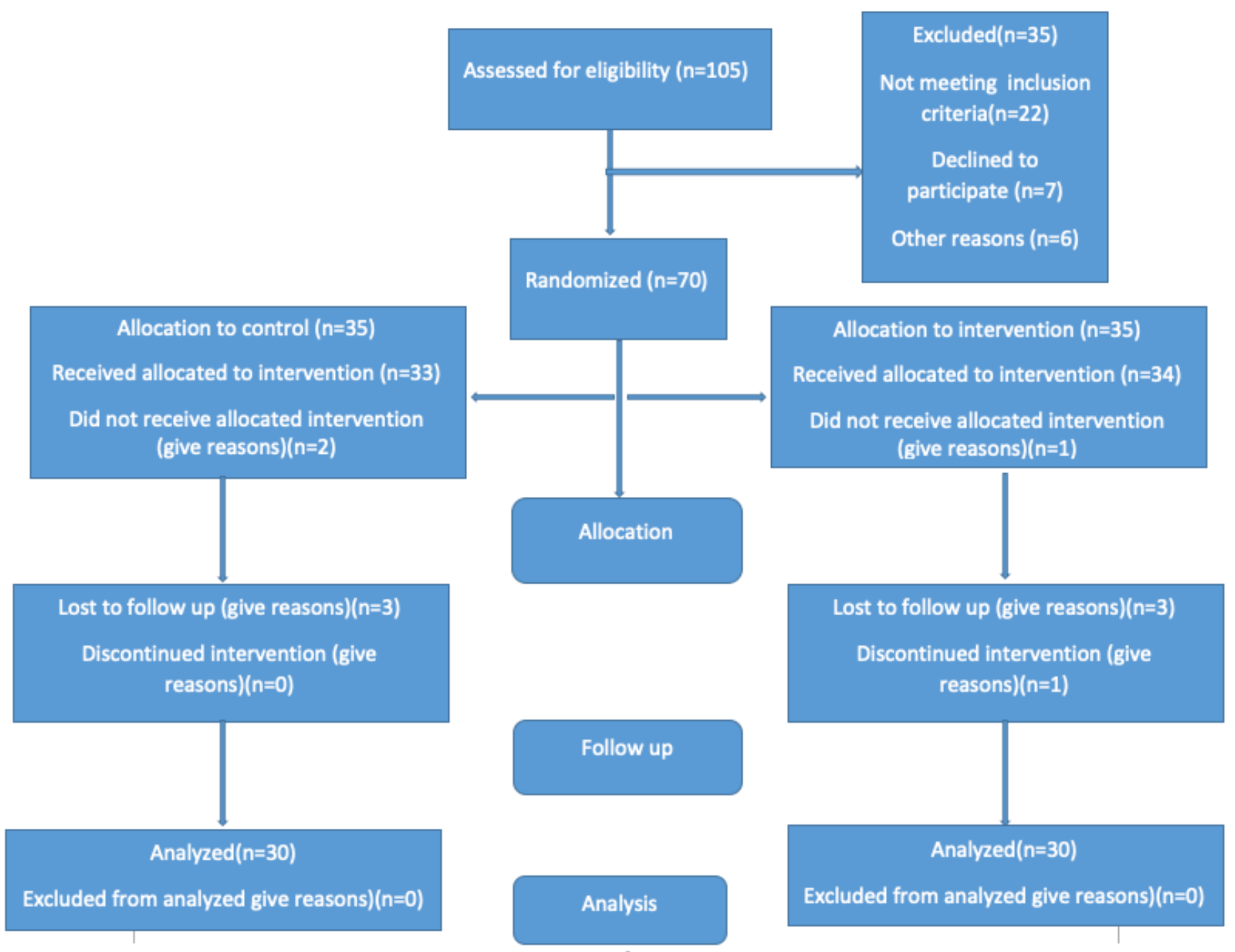

\section{Figure 1}

Checklist 2010 Flow Diagram

\section{Supplementary Files}

This is a list of supplementary files associated with this preprint. Click to download.

- Appendix1.docx 